\title{
Perspective
}

PERSPECTIVE Actualité en histoire de l'art

4 | 2007

Genre et histoire de l'art

\section{Questions de genre et histoire de l'art en Italie}

\section{Laura lamurri}

\section{OpenEdition}

\section{Journals}

Édition électronique

URL : http://journals.openedition.org/perspective/3580

DOI : 10.4000/perspective.3580

ISSN : 2269-7721

Éditeur

Institut national d'histoire de l'art

Édition imprimée

Date de publication : 31 décembre 2007

Pagination : 716-721

ISSN : 1777-7852

\section{Référence électronique}

Laura lamurri, «Questions de genre et histoire de l'art en Italie ». Perspective [En ligne], 4 | 2007, mis en ligne le 31 mars 2018, consulté le 01 octobre 2020. URL : http://journals.openedition.org/perspective/ 3580 ; DOI : https://doi.org/10.4000/perspective.3580 


\title{
Questions de genre et histoire de l'art en Italie
}

\author{
Laura lamurri
}

1 Tout récemment, dans le catalogue de l'exposition WACK!, Judith Russi Kirshner a consacré au féminisme italien un article centré, en ce qui concerne la critique d'art, sur les personnalités de Carla Lonzi, Anne-Marie Sauzeau et Lea Vergine ${ }^{1}$. Bien documenté et argumenté, il souligne la qualité de la réflexion théorique et critique dans la diversité des parcours des trois femmes et paraît approprié pour interroger, parallèlement au propos de l'importante exposition américaine, la question des relations entre féminisme et gender studies: si le rapport entre les deux termes est évident, leur déroulement historique n'a pas toujours été linéaire.

\section{« L'altra metà dell'avanguardia »}

2 Des trois figures majeures identifiées par J. Russi Kirshner, Lonzi et Sauzeau ont joué un rôle de premier plan dans le féminisme italien. Fondatrice avec Carla Accardi d'un des premiers groupes de femmes (Rivolta femminile) en 1970 et de la pratique de l'autoconscience (autocoscienza), C. Lonzi a été aussi l'une des premières théoriciennes de ce qu'on a ensuite nommé la " pensée de la différence ». Bien qu'elle ait abandonné la critique d'art dès 1970, ses textes et ses conversations avec les artistes (hommes et femmes) publiés au cours des années 1960 se caractérisent par une vue singulièrement pénétrante et par une qualité relationnelle $\operatorname{rare}^{2}$ : ce trait personnel, en même temps que sa critique radicale de la culture, paraît fondamental pour les développements successifs des gender studies d'un point de vue à la fois intellectuel et politique. A.M. Sauzeau a elle aussi été engagée dans le mouvement féministe, sans pour autant abandonner la critique d'art. Ses essais, importants du point de vue théorique et d'une lecture fascinante, ont permis une première circulation en langue italienne des thèses de Luce Irigaray, Julia Kristeva et Juliet Mitchell ${ }^{3}$; également publiés en anglais, ils ont atteint un public international grâce aux contacts avec le réseau américain et notamment avec Lucy Lippard ${ }^{4}$. Fondatrice des Edizioni delle donne (avec Manuela 
Fraire, Elisabetta Rasy et Mary Caronia), A.-M. Sauzeau a pris activement part au collectif romain Donne e arte avec d'autres historiennes de l'art telles que Silvia Bordini et Maria Paola Maino.

Bien que Lea Vergine n'ait pas participé au mouvement de libération des femmes, c'est à elle qu'on doit le premier exploit public d'une histoire de l'art qui prenne en compte les femmes artistes de l'avant-garde ou, comme elle le dit elle-même, « Eurydice sans Orphée ». Son exposition L'altra metà dell'avanguardia, itinérante de Milan à Rome et à Stockholm, a connu un grand succès ${ }^{5}$; le catalogue et la documentation - les images sont dues pour la plupart à Maria Mulas - témoignent des difficultés à retrouver les traces d'artistes qui furent «allogènes et métèques » (p.17), âgées au moment de l'exposition et souvent tombées dans l'oubli (Sonia Terk Delaunay, Dora Maar, Greta Knutson et bien d'autres): les notices biographiques de la première édition du catalogue témoignent des efforts déployés pour rassembler quelques éléments sur la vie d'artistes jadis célèbres. Les œuvres racontent une histoire différente de celle que la tradition normative de l'histoire des avant-gardes relatait: si beaucoup de travail restait sans doute encore à faire, le succès même de l'exposition semblait ouvrir un champ d'études nouveau et immense.

4 La recherche de Lea Vergine sur l'avant-garde avait été précédée par un livre de Simona Weller très engagé, consacré à l'art italien $\mathrm{du} \mathrm{xx}^{\mathrm{e}}$ siècle ${ }^{6}$ : première enquête sur les femmes artistes en Italie, articulée en questionnaires et interviews, témoignages et citations, Il complesso di Michelangelo proposait aussi un premier essai de recensement de la présence féminine tout au long $d u x^{e}$ siècle, évaluée à près de 250 artistes dont la plupart étaient presque inconnues ou oubliées en 1976. Les dates sont parfois éloquentes : un an après le livre de $\mathrm{S}$. Weller, dans un volume collectif paru aux éditions Einaudi, on pouvait lire la traduction italienne du célèbre article de Linda Nochlin, Why Have There Been no Great Women Artists ?7 , dont l'importance n'a pas besoin d'être soulignée.

5 Conséquence évidente de l'effervescence du climat politique et social des années 1970, les premières études d'histoire de l'art orientées selon une perspective de genre portent inévitablement sur la contemporanéité. Les Edizioni delle Donne, véritable laboratoire de la culture des femmes en Italie, ont publié à partir de 1976 les traductions du manifeste SCUM de Valerie Solanas, des quelques écrits de Laure [Colette Peignot], du texte de Marina Tsvetaïeva consacré à Natal'ja Goncarova ${ }^{8}$; exception significative dans une sélection évidemment marquée par l'urgence du présent et du passé récent: les actes du procès pour viol qui avait opposé Artemisia Gentileschi à Agostino Tassi, recueillis par Eva Menzio9. Toujours chez les mêmes éditeurs, à partir de 1981, Claudia Salaris avait commencé à rééditer les romans futuristes ${ }^{10}$, pour aboutir en 1982 à son étude, fondamentale pour la recherche, sur les nombreuses femmes artistes et poètes qui ont pris part au mouvement fondé par Filippo Tommaso Marinetti ${ }^{11}$. Bien que l'étude de C. Salaris ait été consacrée à la littérature et soit complétée par une anthologie de textes, les pratiques hybrides du futurisme, et notamment les tables parolibere (pages sous le signe des «mots en liberté » et de la révolution typographique inaugurée par Marinetti), ont contribué à une mise au point de la participation artistique des femmes et au début d'une recherche, parfois impossible $d u$ fait de la perte des témoignages figuratifs, dont l'exposition L'altra metà dell'avanguardia avait donné quelques aperçus ${ }^{12}$. 
6 Les futuristes, dans les années suivantes, sont devenues un objet d'étude passionnant, d'autant plus surprenant dans sa richesse si l'on pense à l'allure très machiste du mouvement et au mépris de la femme proclamé explicitement par Marinetti dans son premier manifeste (1909). À côté des recherches ultérieures de C. Salaris, parues pour la plupart dans des revues, Lia Giachero a publié à partir de 1989 de nombreux essais et articles consacrés surtout à Benedetta Cappa Marinetti, avant d'orienter ses études sur le groupe de Bloomsbury et notamment Vanessa Bell ${ }^{13}$. Les difficultés de la recherche sur les artistes, la perte des œuvres, l'ambiguïté du statut attribué parfois par les artistes elles-mêmes à leur passé futuriste, les complications dues souvent au désir des héritiers d'en finir avec les souvenirs désagréables des mères absentes ${ }^{14}:$ bref les obstacles imposés par un oubli systématique et à différents niveaux ont été la cause d'un certain retard dans le recueil des matériaux et dans l'élaboration d'une étude complète, parue enfin en anglais en 1997 par les soins de Mirella Bentivoglio et Franca Zoccoli $^{15}$.

7 Les vicissitudes des études sur les futuristes et sur les artistes de l'avant-garde en général sont exemplaires des difficultés d'une recherche qui a affaire à la perte de mémoire, ou pour mieux dire à une coupure dans la transmission de la mémoire qui empêche le passage de celle-ci à l'histoire. Cette démarche inégale et discontinue est symptomatique de l'histoire des gender studies en Italie et de ses liens avec l'histoire du féminisme. Avec les publications des Edizioni delle Donne et les réflexions théoriquement approfondies de certains collectifs, et surtout après le succès de l'exposition L'altra metà dell'avanguardia, on aurait facilement pu envisager une rapide floraison de la recherche; cependant, les choses ne se sont pas passées de cette façon.

$8 \mathrm{Au}$ contraire, hormis quelques exceptions remarquables, les études ont connu une période assez longue d'impasse et il a fallu attendre la moitié des années 1990 pour une pleine reprise des travaux. Par rapport à ce qui s'est passé dans les pays anglo-saxons notamment, en Italie les gender studies dans le domaine des arts visuels et de l'histoire de l'art sont restés pendant longtemps l'affaire des différentes personnalités des chercheuses qui, bien que liées entre elles par des relations personnelles, ont beaucoup travaillé dans l'ombre, sans que soit assurée une circulation de leurs travaux. Pour des raisons différentes, liées souvent aux caractères propres de la réflexion critique à l'intérieur du mouvement de libération des femmes, mais aussi bien aux résistances des institutions, en Italie ces études n'ont pris ni l'habitus, ni la structure traditionnelle du savoir universitaire (enseignements spécifiques et départements, groupes de recherche, doctorats, bibliothèques, musées). Cette distance par rapport aux institutions - plus ou moins cherchée, plus ou moins forcée - a permis sans doute de maintenir l'horizontalité des pratiques chères au féminisme, hors des hiérarchies du pouvoir académique ; mais cela a provoqué aussi une carence qui a eu ses conséquences dans la possibilité même de poursuivre, voire d'encourager ces études auprès des nouvelles générations de chercheurs. Situation qui continue singulièrement dans une certaine mesure à affecter l'histoire de l'art, tandis que dans les champs historique et littéraire on a pu constater un développement significatif ces dix dernières années.

\section{À la recherche des femmes artistes du passé}

9 Les conditions académiques ne sont pas étrangères au petit nombre de publications et de monographies au sujet des peintres de l'époque moderne. Si l'on connaît les 
difficultés de la recherche sur le Moyen Âge, liées entre autres aux pratiques d'atelier et à l'impossibilité de donner parfois une identité aux artistes, la Renaissance et le Baroque ont été l'objet d'études éparses, souvent encouragées par des publications en langue anglaise. L'exemple d'Artemisia Gentileschi est intéressant: après le roman d'Anna Banti ${ }^{16}$ et l'édition des actes du procès citée plus haut, un regain d'intérêt au début des années 1990 a été la conséquence de la monographie de Mary Garrard, parue en 1989 et inégalement commentée dans les revues italiennes ${ }^{17}$; après une exposition en 1991, on a pourtant attendu 1998 pour une monographie, publiée par Tiziana Agnati et Francesca Torres dans la série L'altra metà dell'arte, consacrée aux femmes artistes, des éditions Selene ${ }^{18}$. Une histoire aussi accidentée se repère aussi dans le sort critique de peintres telles que Sofonisba Anguissola, Elisabetta Sirani, Lavinia Fontana, pour rappeler seulement les plus célèbres : en ce qui concerne Sofonisba Anguissola, la première grande exposition itinérante a été organisée en $1994^{19}$; Elisabetta Sirani a bénéficié de quelques attentions à partir de la fin du xix siècle du fait de sa mort par empoisonnement, mais sa bibliographie comporte un vide de 1904 à l'exposition de 2004, interrompu seulement par deux communications parues dans des revues en 1949 et en $1976^{20}$. Lavinia Fontana a fait l'objet des travaux de Vera Fortunati et Maria Teresa Cantaro à partir de $1986^{21}$. À l'inverse, le cas de Rosalba Carriera est particulier pour l'intérêt constant que l'artiste a suscité, même en dehors de toute perspective de genre, mais son caractère exceptionnel est mis en évidence par l'absence presque totale d'études sur le XvIII ${ }^{\mathrm{e}}$ siècle jusqu'à la moitié des années $1990^{22}$.

Des études sur les contextes historiques, poussées par l'idée d'une attention aux milieux et aux connexions plutôt qu'aux personnalités exceptionnelles, ont connu le rythme discontinu des gender studies, l'accord et les conflits avec la contemporanéité historique et politique, avec parfois un surcroît de méfiance par rapport aux études qui trahissent le genre codifié de la monographie. Et pourtant, notamment en l'absence de figures de « grandes » - comme le disait Linda Nochlin - artistes, les recherches sur les milieux sont d'autant plus décisives pour comprendre les réseaux, les relations, la transmission du savoir, les pratiques et les organisations des femmes. Il faut donc rappeler l'exposition milanaise Dal salotto agli ateliers qui, à la fin des années 1980, a constitué un premier essai de présentation, à travers les œuvres, du parcours complexe depuis la pratique en amateur jusqu'à la professionnalisation des artistes, observé dans le cadre du laboratoire de la modernité italienne que fut Milan entre la fin du XIX et le début du $\mathrm{Xx}^{\mathrm{e}}$ siècle $^{23}$. Des travaux semblables ont été menés dans le domaine du design, avec les études de Maria Paola Maino, Irene de Guttry et Anty Pansera, et dans le cadre de la Biennale Donna de Ferrare, qui depuis 1984 propose souvent des ensembles thématiques à côté d'expositions historiques ${ }^{24}$.

\section{La situation actuelle : richesse et ambiguïtés}

11 On a déjà entrevu que les gender studies ont pris une orientation différente dans la seconde moitié des années 1990. En coïncidence sans doute avec certaines tendances de l'art contemporain caractérisé, en Italie comme dans le reste du monde occidental, par une présence féminine de jour en jour plus imposante du point de vue quantitatif et plus importante pour le caractère particulièrement marquant des œuvres; ou bien à cause d'une nouvelle génération de chercheuses, qui ont fréquenté les textes anglosaxons et s'efforcent de reconstruire une généalogie du savoir différente. Dans son 
projet d'un Dizionario della cultura femminile (sous la direction d'Annarita Buttafuoco, Maria Gregorio, Katie Roggero), Patrizia Zambrano avait entamé en 1997 la réalisation d'une vaste section Arte mettant à contribution un grand nombre de chercheuses de générations diverses engagées dans une recherche sur différents sujets, des artistes aux commanditaires, des historiennes aux galeristes, avec des articles thématiques faisant le lien entre les entrées monographiques : un projet encyclopédique qui aurait fourni un panorama, inédit en langue italienne, des recherches dans le domaine des gender studies. Même si le dictionnaire est resté pour l'instant inachevé, P. Zambrano a le mérite d'avoir constitué un réseau de chercheuses qui continuent entre autres à travailler dans cette voie ${ }^{25}$.

L'insouciance ou la sous-estimation de plusieurs éditeurs italiens à l'égard des gender studies a produit un retard manifeste dans la circulation des textes publiés à l'étranger ; on doit à Maria Antonietta Trasforini, sociologue de la culture, la traduction de l'essai fondamental de Griselda Pollock, Modernity and the Spaces of Femininity, paru avec The Artist and the Flâneur de Janet Wolff et d'autres écrits importants, dont certains inédits, dans le livre Arte a parte (2000). De là l'auteure a poursuivi ses études sur les enjeux et les conditions sociales dans lesquelles les femmes ont négocié leur espace professionnel, en tant qu'artistes, dans la modernité ${ }^{26}$. Contemporanee, publié en 2000 par Emanuela De Cecco et Gianni Romano, a mis à la disposition des lecteurs italiens beaucoup de textes critiques sur nombre d'artistes contemporaines de la scène internationale, sans oublier les artistes italiennes: on en dégage l'image d'un art globalisé quoique animé de singularités puissantes, où les femmes ont gagné un peu partout une position centrale et enfin abondamment reconnue.

On ne saurait mesurer à quel point le changement de statut des femmes artistes contemporaines a contribué d'une part à la redécouverte de certaines artistes vivantes, d'autre part au développement des études historiques, surtout par rapport aux XIX et $\mathrm{xx}^{\mathrm{e}}$ siècles. En 2001, sur commande du Ministero dei Beni Culturali, Carolina Brook, Laura Iamurri et Sabrina Spinazzè se sont chargées de l'organisation d'un colloque intitulé Donne e arti visive nell'Italia del Novecento (2001) qui fut, dans sa nécessaire partialité et à travers les apports des expertes (la plupart desquelles déjà nommées dans cet article), le premier regard d'ensemble sur la présence féminine dans les arts du xx siècle ; en 1998, à Turin, avait déjà été tentée une mise au point sur un domaine spécifiquement contemporain, la photographie ${ }^{27}$.

Ces dernières années, les publications et les expositions consacrées aux femmes se sont multipliées et il est impossible d'en donner un tableau exhaustif, même si nombre de textes fondamentaux restent à traduire et ne circulent pour l'instant que dans les milieux directement intéressés à ce genre de recherche. Un nombre important d'études marquées par l'approche gender, a été publié, parmi lesquelles il faut signaler les recherches de Silvia Bordini sur l'art électronique ou les réflexions de Donatella Franchi sur la pensée et les pratiques des femmes artistes ${ }^{28}$. On a commencé à publier les écrits des artistes, à reconnaître la place due aux artistes vivantes, à en écrire l'histoire ${ }^{29}$, parfois à reconsidérer des périodes restées longtemps marquées exclusivement par des études consacrées aux hommes artistes, et à publier des histoires de femmes artistes ${ }^{30}$ (pas toujours, à vrai dire, à partir d'une réelle optique de genre: aujourd'hui le risque est grand de prendre les gender studies pour une simple mode ou pire, pour l'exploitation de nouveaux secteurs du marché de l'art). Enfin, dans le cadre d'une conscience accrue de l'histoire des femmes, on essaie de retisser les fils 
des pratiques savantes des générations précédentes, grâce à l'aide des historiennes, à la recherche sur les sources, aux témoignages directs qui permettent de combler les lacunes de la mémoire.

\section{NOTES}

1. Judith Russi Kirshner, "Voices and Images of Italian Feminism ", dans WACK! Art and the Feminist Revolution, Cornelia Butler éd., (cat. expo., Los Angeles, MOCA/Washington, National Museum of the Women in the Arts/New York, PS1/Vancouver, Vancouver Art Gallery), Cambridge/Londres, 2007, p. 384-399.

2. Voir notamment Carla Lonzi, Autoritratto, Bari 1969. Voir aussi Maria Luisa Boccia, L'io in rivolta. Vissuto e pensiero di Carla Lonzi, Milan, 1990 ; Laura Iamurri, « 'Un mestiere fasullo' : note su Autoritratto di Carla Lonzi ", dans Maria Antonietta Trasforini éd., Donne d'arte. Storie e generazioni, Rome, 2006, p. 115-132.

3. Anne-Marie Sauzeau Boetti, «Lo specchio ardente: appunti teorici sul concetto di 'altra creatività', di segno (o gene ?) femminile », dans Data, 16-17, 1975.

4. Sauzeau Boetti, «Negative Capability as Practice in Women's Art ", dans Studio International, 191, 1976.

5. L'altra metà dell'avanguardia, 1910-1940, Lea Vergine éd., (cat. expo. Milan, Palazzo Reale/Rome, Palazzo delle Esposizioni/Stockolm, Kulturhuset), Milan 1980, (rééd. Milan 2005).

6. Simona Weller, Il complesso di Michelangelo. Ricerca sul contributo dato dalla donna all'arte italiana del '900, Macerata, 1976.

7. Paru originairement dans Art News, 69/9, 1970, p. 22-39 et 67-71), l'article de Linda Nochlin a été réédité dans Vivian Gornick, Barbara K. Moran éd., Woman in Sexist Society, New York/ Londres, 1971, traduit en italien sous le titre La donna in una società sessista. Potere e dipendenza, Turin, 1977 ; trad. fr. : «Pourquoi n'y-a-t-il pas eu de grandes artistes femmes?», dans Linda Nochlin, Femmes, art et pouvoir et autres essais, Nîmes, 1993, p. 201-244.

8. Valerie Solanas, S.C.U.M.: Manifesto per l'eliminazione dei maschi, Anne-Marie Boetti éd., Rome, 1976 ; Laure (Colette Peignot), Storia di una ragazzina e altri scritti, Rome, 1976 ; Marina Ivanovna Tsvetaïeva, Natal'ja Goncarova : ritratto di un'artista, Luciana Montagnani éd., Milan, 1982.

9. Eva Menzio éd., Atti di un processo per stupro. Artemisia Gentileschi, Agostino Tassi, Milan, 1981.

10. Rosa Rosà, Una donna con tre anime: romanzo futurista, Claudia Salaris éd., Milan, 1981.

11. Claudia Salaris, Le futuriste, Donne e letteratura d'avanguardia in Italia (1909-1944), Milan, 1982.

12. Voir aussi Claudia Salaris, «Donne nel Futurismo dal 1920 al 1944 », dans I luoghi del Futurismo (1909-1944), (colloque, Macerata, 1982), Rome, 1986, p. 193-204. Sur les difficultés de la recherche, voir C. Salaris, « Incontri con le futuriste ", dans Laura Iamurri, Sabrina Spinazzè éd., L'arte delle donne nell'Italia del Novecento, (colloque, Rome, 2001), Rome, 2001, p. 50-66.

13. Lia Giachero, "Benedetta nel clima del futurismo romano", dans Casa Balla e il futurismo a Roma, Enrico Crispolti éd., (cat. expo., Rome, Villa Medicis, 1989), Rome 1989, p. 409-419 ; « '... Grands étalages/de mers et de nuages...' Due inediti bozzetti per vetrate di Benedetta Marinetti », dans Ricerche di Storia dell'Arte, 40, 1990, p. 81-86; "I capricci della memoria : riflessioni sulle futuriste ", dans Maria Antonietta Trasforini éd., Arte a parte. Donne artiste fra margini e centro, Milan, 2000, p. 133-144.

14. Sur ce point, voir Claudia Salaris dans Iamurri, Spinazzè, 2001, cité n. 12. 
15. Mirella Bentivoglio, Franca Zoccoli, The Women Artists of Italian Futurism. Almost lost to history, New York, 1997. Voir aussi Franca Zoccoli, Benedetta Cappa Marinetti. L'incantesimo della luce, Milan, 2000. Mirella Bentivoglio est aussi à mentionner pour Materializzazione del linguaggio, section de la Biennale de Venise de 1978 (cat. expo., Venise, Magazzini del sale alle Zattere), Venise, 1978.

16. Anna Banti, Artemisia : romanzo, Florence, 1947, avec de nombreuses rééditions ; trad. fr.: Artemisia, Paris, 1989.

17. Mary D. Garrard, Artemisia Gentileschi : the image of the female hero in Italian baroque art, Princeton, 1989.

18. Artemisia, Roberto Contini, Gianni Papi éd., (cat. expo., Florence, Casa Buonarroti, 1991), Rome, 1991 ; Tiziana Agnati, Francesca Torres, Artemisia Gentileschi, Milan, 1998.

19. Sofonisba Anguissola e le sue sorelle, Mina Gregori éd., (cat. expo., Cremona, Centro Culturale S. Maria della Pietà, 1994/Vienne, Kunsthistorisches Museum, 1995/Washington, National Museum of the Women in the Arts), Rome, 1994.

20. Antonio Manaresi, Il processo di avvelenamento fatto nel 1665-66 in Bologna contro Lucia Tolomelli per la morte di Elisabetta Sirani, Bologne, 1904; Fernando Ghedini, "Due Sibille di Elisabetta Sirani », dans Atti e memorie dell'Accademia Clementina, 4, 1950, p. 85-86 ; Paolo Bellini, « Elisabetta Sirani », dans Nouvelles de l'estampe, 30, 1976, p. 7-12 ; Elisabetta Sirani : "pittrice eroina » 1638-1665, Jadranka Bentini, Vera Fortunati éd., (cat. expo, Bologne, Museo Civico, 2004), Bologne, 2004 ; voir aussi Adelina Modesti, Elisabetta Sirani : una 'virtuosa' del Seicento bolognese, Bologne, 2004.

21. Vera Fortunati, «Lavinia Fontana », dans Pittura bolognese del ‘500, Bologne, 1986, p. 727-737 ; Maria Teresa Cantaro, Lavinia Fontana bolognese, " pittora singolare », 1552-1614, Milan, 1989 ; Lavinia Fontana, 1552-1614, Vera Fortunati éd., (cat. expo., Bologne, Museo Civico Archeologico, 1994), Milan, 1998 ; Lavinia Fontana, 1552-1614, Vera Fortunati éd., (cat. expo., Washington, National Museum of the Women in the Arts, 1998), Milan, 1998.

22. Voir Caterina Limentani Virdis éd., "Le tele svelate " : antologia di pittrici venete dal Cinquecento al Novecento, Mirano, 1996. À propos de Rosalba Carriera, à côté de l'édition des écrits (Bernardina Sani éd., Rosalba Carriera. Lettere, diari, frammenti, Florence, 1985), voir le tout récent Rosalba Carriera: prima pittrice d'Europa, Giuseppe Pavanello éd., (cat. expo., Venise, Galleria di Palazzo Cini a San Vio 2007), Venise, 2007.

23. Dal salotto agli ateliers : produzione artistica femminile a Milano, 1880-1920, Aurora Scotti, Maria Teresa Fiorio éd., (cat. expo., Milan, Museo Civico), Milan, 1989.

24. Pour une table récapitulative des douze éditions de la Biennale Donna de Ferrare, voir le site : http://www.artecultura.fe.it. L'édition de 2002 était consacrée au design : X Biennale Donna. Dal merletto alla motocicletta: artigiane/artiste e designer nell'Italia del Novecento, Anty Pansera éd., (cat. expo., Ferrare, Padiglione d'Arte Contemporanea), Cinisello Balsamo, 2002. Voir aussi Il design delle donne, Nicoletta Livi Bacci et al. éd., (cat. expo., Florence, Libreria delle Donne, 1993) Milan, 1991.

25. Sans pouvoir mentionner toutes les auteures associées au projet, je voudrais rappeler au moins: Silvia Bignami, Silvia Bordini, Giovanna Capitelli, Francesca Castellani, Daniela De Angelis, Cristina De Benedictis, Sara Fontana, Valentina Furlanetto, Laura Iamurri, Laura Lombardi, Maria Grazia Messina, Ilaria Miarelli Mariani, Paola Pallottino, Caterina Volpi.

26. Maria Antonietta Trasforini, Nel segno delle artiste. Donne, professioni d'arte e modernità, Bologne, 2007 ; Trasforini, 2000, cité n. 13 ; voir aussi Trasforini, 2006, cité n. 2.

27. Nicoletta Leonardi éd., L'altra metà dello sguardo. Il contributo delle donne alla storia della fotografia, (colloque, Turin, 1998), Turin, 2001 ; Iamurri, Spinazzè, 2001, cité n. 12.

28. Silvia Bordini, Arte elettronica, Florence, 2004 ; Donatella Franchi éd., Matrice. Pensiero delle donne e pratiche artistiche, Milan, 2004. Voir aussi, sur un sujet sensible comme celui de l'autoreprésentation, autobiografia/autoritratto, Laura Iamurri éd., (cat. expo., Rome, Museo H.C. Andersen, 2007), Rome 2007. 
29. Pour rappeler seulement les cas plus fameux, Bice Lazzari a été l'objet des recherches de Monica Naldi («Bice Lazzari: il percorso fra arte applicata e astrattismo negli anni della formazione [1916-1934]», dans Ricerche di Storia dell'Arte, 57, 1995, p. 57-67), et Sergio Cortesini (Bice Lazzari, l'arte come misura : ritratto di una pittrice tra Venezia e Roma, Rome, 2002). Carol Rama a connu un regain d'intérêt, dont ont témoigné diverses expositions ; la Galleria Nazionale d'Arte Moderna a consacré une exposition à Elisa Montessori ; une rétrospective de Titina Maselli (décédée en 2005) est prevue à Rome pour 2008; on attend encore une grande exposition sur Carla Accardi. Les écrits de Ketty La Rocca ont été publiés par Lucilla Saccà (Turin, 2005).

30. Simona Bartolena, Arte al femminile. Donne artiste dal Rinascimento al XXI secolo, Milan, 2003 ; Martina Corgnati, Artiste. Dall'impressionismo al nuovo millennio, Milan, 2004 ; A.i. 20 : artiste italiane nel ventesimo secolo, Elena Lazzarini, Pier Paolo Pancotto éd., (cat. expo., Seravezza, Palazzo Mediceo), Prato, 2004 ; Pier Paolo Pancotto, Artiste a Roma nella prima metà del 900, Rome, 2006.

\section{INDEX}

Keywords : Italian feminism, historiography, gender studies, futurism

Mots-clés : féminisme italien, féminisime, historiographie, gender studies, futurisme

Index géographique : Italie

Index chronologique : 1900, 2000

\section{AUTEUR}

\section{LAURA IAMURRI}

Università degli Studi Roma Tre 